\title{
Doenças neurológicas em cães atendidos no Hospital Veterinário da Universidade Federal de Santa Maria, RS: 1.184 casos $(2006-2013)^{1}$
}

\author{
Rafael Oliveira Chaves ${ }^{2 *}$, Diego Vilibaldo Beckmann ${ }^{3}$, Rosmarini Passos dos \\ Santos ${ }^{2}$, Graciane Aiello², Amanda Oliveira Andrades², Raquel Baumhardt', \\ Laís Barbieri Silveira ${ }^{4}$ e Alexandre Mazzanti ${ }^{5}$
}

\begin{abstract}
Chaves R.O., Beckmann D.V., Santos R.P., Aiello G., Andrades A.O., Baumhardt R., Silveira L.B. \& Mazzanti A. 2014. [Neurological diseases in dogs examined at the Veterinary Teaching Hospital of the Federal University of Santa Maria, RS: 1.184 cases (2006-2013).] Doenças neurológicas em cães atendidos no Hospital Veterinário da Universidade Federal de Santa Maria, RS: 1.184 casos (2006-2013). Pesquisa Veterinária Brasileira 34(10):996-1001. Departamento de Clínica de Pequenos Animais, Universidade Federal de Santa Maria, Avenida Roraima 1000, Camobi, Santa Maria, RS 97105-900, Brazil. E-mail: rafaelochaves@hotmail.com

A retrospective study including dogs with neurological disease was conducted at the Service of Neurology (SN) of the Veterinary Teaching Hospital, Universidade Federal de Santa Maria (UFSM) from 2006 to 2013, with the objective to identify and characterize age, breed, sex and to neurological diseases, and classify them accordingly to the anatomical region and DINAMIT-V acronym. There were evaluated 1,277 neurological records of dogs and obtained the information for inclusion in the study in 1,184 of them being the diagnosis confirmed in $525(44.4 \%)$ and presumptive in 659 dogs (55.6\%). The most common breed was Dachshund (28.7\%), followed by mixed breed. The most affected sites were the spinal cord between T3-L3 (40.9\%) and thalamus-cortex (17.5\%). Most dogs were diagnosed with degenerative disorders (49\%), being intervertebral disk disease the most observed, followed by inflammatory/infectious diseases (16.6\%). It can be concluded that the higher prevalence of neurological disorders in dogs involve the spinal cord and thalamus-cortex, with the most frequent being degenerative and the data obtained may assist future studies associated with frequency and distribution of the main neurological diseases in dogs.
\end{abstract}

INDEX TERMS: Neurology, neurological diseases, neuroanatomical localization, dogs.

RESUMO.- Foi realizado um estudo retrospectivo de cães atendidos no Serviço de Neurologia (SN) do Hospital Veterinário Universitário (HVU) da Universidade Federal de Santa Maria (UFSM), de 2006 a 2013, com o objetivo de identificar

\footnotetext{
${ }^{1}$ Recebido em 12 de março de 2014.

Aceito para publicação em 11 de agosto de 2014

${ }^{2}$ Programa de Pós-Graduação em Medicina Veterinária, área de concentração em Cirurgia, Centro de Ciências Rurais (CCR), Universidade Federal de Santa Maria (UFSM), Camobi, Santa Maria, RS 97105-900, Brasil. *Autor para correspondência: rafaelochaves@hotmail.com

${ }^{3}$ Universidade Federal do Pampa, campus Uruguaiana, BR-472 Km 592, Uruguaiana, RS 97500-970, Brasil

${ }^{4}$ Curso de Medicina Veterinária, CCR-UFSM, Santa Maria, RS.

${ }^{5}$ Departamento de Clínica de Pequenos Animais, CCR-UFSM, Santa Maria, RS.
}

e caracterizar a idade, a raça, o sexo e as doenças neurológicas e classificá-las de acordo com a região anatômica e o acrônimo DINAMIT-V. Foram avaliadas 1.277 fichas neurológicas de cães e obtidas as informações para inclusão no estudo em 1.184 delas, sendo o diagnóstico confirmado em 525 cães $(44,4 \%)$ e presuntivo em 659 (55,6\%). A raça mais frequente foi Dachshund (28,7\%), seguida dos cães sem raça definida. Os locais mais afetados foram medula espinhal entre T3-L3 (40,9\%) e tálamo-córtex (17,5\%). A maioria dos cães foi diagnosticada com doença degenerativa (49\%), sendo a doença do disco intervertebral a mais observada, seguida das doenças inflamatórias/infecciosas (16,6\%). Pode se concluir que a maior prevalência das doenças neurológicas de cães envolve a medula espinhal e o tálamo-córtex, sendo as degenerativas as mais frequentes e os dados obtidos 
podem auxiliar em futuros estudos sobre a frequência e a distribuição das principais doenças neurológicas em cães.

TERMOS DE INDEXAÇÃO: Neurologia, doenças neurológicas, localização neuroanatômica, caninos.

\section{INTRODUÇÃO}

Os trabalhos sobre enfermidades neurológicas geralmente consistem em relatos de casos ou estudos retrospectivos de uma determinada doença (Foster et al. 1988, Heidner et al. 1991, Kipperman et al. 1992, Bagley et al. 1999, Garosi et al. 2005, Snyder et al. 2006) e poucos são aqueles demonstrando as principais doenças neurológicas em cães (Fluehmann et al. 2006, Pellegrino et al. 2011). Uma análise retrospectiva sobre enfermidades neurológicas em felinos foi realizada e a categoria das enfermidades inflamatórias e neoplásicas foram as que prevaleceram, sendo a peritonite infecciosa felina e o linfoma as doenças mais encontradas, respectivamente (Bradshaw et al. 2004). Esta informação obtida foi relevante por gerar uma lista de diagnósticos diferenciais em cães com distúrbios neurológicos (Pellegrino et al. 2011).

No entanto, poucos estudos descreveram a prevalência de efermidades neurológicas em cães e gatos (Bradshaw et al. 2004, Fluehmann et al. 2006, Pellegrino et al. 2011) e geralmente estão confinadas a uma região neuroanatômica, como por exemplo, a medula espinhal (Marioni-Henry et al. 2004) ou de uma doença específica, como por exemplo, a doença de disco intervertebral (Brisson 2010) ou neoplasmas espinhais (Marioni-Henry et al. 2008).

Além disso, dados sobre variações geográficas que afetam a prevalência de doenças neurológicas são limitados. Alguns estudos sobre neoplasmas encefálicos relataram que os tumores mais comuns em cães no leste dos Estados Unidos foram meningiomas (Foster et al. 1988, Heidner et al. 1991), já em outros estudos, envolvendo hospitais de várias regiões desse mesmo país, o astrocitoma foi o mais prevalente (Hayes et al. 1975). Essas diferenças podem ser reflexo de mudanças na prevalência de tumores, diferenças na população de estudo de acordo com as raças ou outras razões desconhecidas (Bagley et al. 1993). Outras doenças ocorrem com maior frequência em determinadas regiões geográficas, como a cinomose, que é mais comum na América Latina do que nos Estados Unidos ou Europa. Sua prevalência varia mesmo entre cidades ou regiões diferentes dentro de um país (Pellegrino et al. 2011).

Devido à escassez de dados epedimiológicos sobre as doenças que afetam o sistema nervoso em cães no Brasil, o objetivo do presente trabalho foi identificar cães com afecções neurológicas atendidos no Serviço de Neurologia de um Hospital Veterinário Universitário na região central do Rio Grande do Sul no período de janeiro de 2006 a agosto de 2013 e obter informações a respeito da idade, do sexo, da raça e classificá-las de acordo com a região anatômica envolvida e classificação das doenças por meio das categorias incluídas no acrônimo DINAMIT-V.

\section{MATERIAL E MÉTODOS}

Foram revisados os arquivos do serviço de neurologia do HVU da UFSM no período de janeiro de 2006 a agosto de 2013 e incluídos no estudo cães que apresentavam história clínica, exame clínico e neurológico de doença neurológica e diagnóstico confirmado por meio de exames complementares, cirurgia ou necropsia, ou diagnóstico presuntivo, quando a suspeita clínica não pôde ser confirmada. Os cães foram distribuídos em cinco grupos etários: menor que um ano de idade, entre um e três anos, entre três e seis anos, entre seis e nove anos e maior que nove anos. Foi realizado exame neurológico para localização das lesões, que foram distribuídas em tálamo-córtex, tronco encefálico, cerebelo, medula espinhal, nervos periféricos, neuromuscular e multifocal (sinais clínicos em mais de uma região neuroanatômica). A localização de lesão na medula espinhal foi definida em C1-C5, C6-T2, T3-L3 e L4-S3. A avaliação neurológica dos cães incluiu: 1) observações gerais (nível de consciência e comportamento), 2) análise da postura e marcha, 3) avaliação das reações posturais (posicionamento proprioceptivo, teste do salto e hemi-salto), 4) avaliação de nervos cranianos, 5) reflexos segmentares espinhais e 6) nocicepção (dor profunda). Como critério de inclusão, os cães ainda necessitavam apresentar, no mínimo, um sinal neurológico condizente com a região anatômica predeterminada no estudo, ou seja, tálamo-córtex, tronco encefálico, cerebelo, medula espinhal, nervo periférico e neuromuscular (Dewey 2006, Lorenz et al. 2011).

A partir do diagnóstico neuroanatômico foi estabelecida uma lista de diagnósticos diferenciais de doenças que poderiam afetar a região anatômica em questão, classificando-as em categorias inseridas no acrônimo DINAMIT V, ou seja, degenerativas (D), doenças inflamatórias/infecciosas (I), neoplásicas ou nutricionais $(\mathrm{N})$, anomalias de desenvolvimento (congênitas) (A), metabólicas (M), idiopáticas (I), traumáticas ou tóxicas (T) e vascular (V) (Fluehmann et al. 2006, Da Costa \& Moore 2010, Pellegrino et al. 2011).

Em seguida, foram selecionados os métodos de diagnóstico complementares específicos para identificar a provável etiologia (diagnóstico etiológico) e a extensão da lesão (Dewey 2006). Em todos os casos foram solicitados hemograma, bioquímica sérica (determinação de ureia, creatinina, glicose, alanina aminotransferase [ALT], fosfatase alcalina [FA], proteína sérica total e albumina) e urinálise. Em cães com suspeita de doenças neuromusculares foi realizada a determinação de creatinofosfoquinase (CPK) e asparato aminotranferase (AST). Em alguns destes animais foi realizada biopsia muscular seguida de análise histológica. Na suspeita de doenças metabólicas como hipotireoidismo, a função tireoidiana foi avaliada pela determinação de T4 total, T4 livre em diálise e hormônio tireotrófico (TSH). De acordo com as necessidades de diagnóstico, em alguns indivíduos foi realizada análise de líquido cerebroespinhal (LCE), mediante punção na cisterna magna e/ou lombar (L5-L6). Os exames de imagem utilizados foram radiografia simples e contrastada (mielografia), ultrassom, e em alguns casos, tomografia computadorizada (TC). Outros diagnósticos etiológicos foram confirmados por cirurgia ou necropsia e histopatologia.

O diagnóstico presuntivo foi estabelecido com base no histórico, no exame neurológico, na progressão da doença, na resposta ao tratamento e na exclusão de outras afecções por meio de exames complementares (Fluehmann et al. 2006). Para casos de óbito sem confirmação do diagnóstico por exames pós-mortais foi mantida a suspeita e os casos classificados de acordo com o diagnóstico presuntivo. Cães encaminhados para necropsia foram submetidos à eutanásia por opção dos proprietários ou morreram em decorrência da doença.

\section{RESULTADOS E DISCUSSÃO}

No período de sete anos e oito meses foram encaminhados 1277 cães (média de 166 casos por ano) com suspeita de 
alterações neurológicas ao Serviço de Neurologia da Instituição. Desse total, em 32 cães $(2,5 \%)$ não foi possível realizar o diagnóstico por falta de informações nos registros avaliados, o que ocorreu em pacientes atendidos no ano de 2006, quando o Serviço de Neurologia estava no início e o banco de dados não era atualizado diariamente e, em 61 casos $(4,8 \%)$, não foram encontradas alterações neurológicas por serem basicamente afecções do sistema locomotor. Fluehmann et al. (2006) realizaram um estudo retrospectivo com 4.497 cães (média de 375 casos por ano) com enfermidades neurológicas num período de doze anos e Pellegrino et al. (2011) avaliaram 1.652 cães (média de 708 casos por ano) em um período de dois anos e três meses.

A distribuição etária neste estudo variou de um mês a 23 anos, com média de seis anos e dois meses de idade. Do total de 1.184 cães com doença neurológica, em $21(1,8 \%)$, a idade não constava nos registros. Dos 1.163 cães que tiveram a idade informada, $106(9,1 \%)$ tinham menos de um ano, $126(10,8 \%)$ entre um e três anos, 313 $(26,9 \%)$ entre três e seis anos, $344(29,6 \%)$ entre seis e nove anos, $274(23,6 \%)$ tinham mais de nove anos de idade (Fig.1). Dos 1.184 cães com diagnóstico de doença neurológica, em sete $(0,59 \%)$ o sexo não estava informado nos registros. Dos 1.177 cães com sexo especificado nos registros, 612 (52\%) eram fêmeas e 565 (48\%) eram machos (Fig.1), ou seja, uma relação macho:fêmea de 0,92. Nos trabalhos de Fluehmann et al. (2006) (57,8\% machos e $41,3 \%$ fêmeas) e Pellegrino et al. (2011) (54\% machos e $46 \%$ fêmeas), machos foram pouco mais prevalentes que fêmeas.

Dos 1.176 cães que tiveram a raça informada, 312 $(26,5 \%)$ não tinham raça definida e $864(73,5 \%)$ eram de raça pura, ou seja, uma relação entre cães sem raça definida e cães de raça pura de 0,36 . Cães de 45 raças diferentes tinham sinais neurológicos e, dentre os 1.176, os que tinham uma distribuição de frequência igual ou superior a $1 \%$ do total incluíram: Dachshund (28,7\%), Poodle $(8,4 \%)$, Pinscher $(4,4 \%)$, Yorkshire terrier $(3,8 \%)$, Boxer $(3,6 \%)$, Cocker spaniel inglês (2,2\%), Rottweiler $(1,7 \%)$, Lhasa apso $(1,6 \%)$, Labrador retriever $(1,4 \%)$, Pastor alemão $(1,4 \%)$, Beagle $(1,4 \%)$, Maltês $(1,2 \%)$, Shih tzu $(1,1 \%)$ e

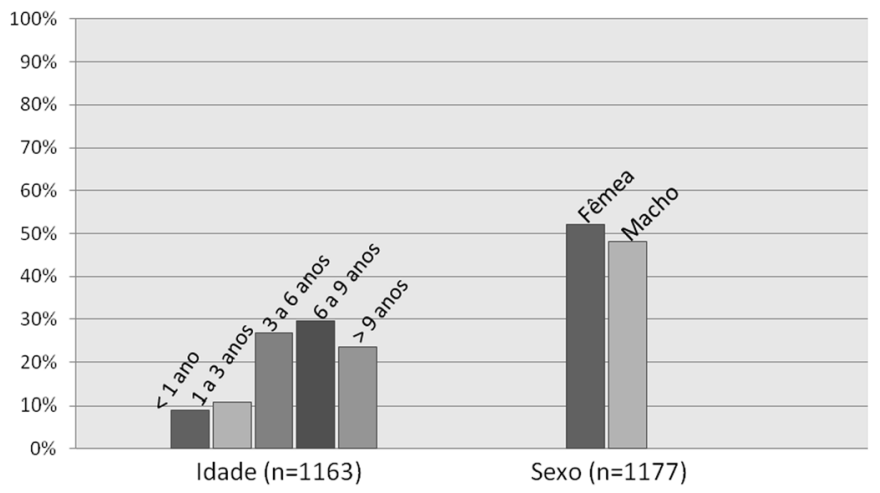

Fig.1. Distribuição de frequências dos grupos de idade (n=1163) e sexo $(n=1170)$ de cães atendidos no Serviço de Neurologia do Hospital Veterinário Universitário da Universidade Federal de Santa Maria no período de 2006 a 2013, diagnosticados com doença neurológica.

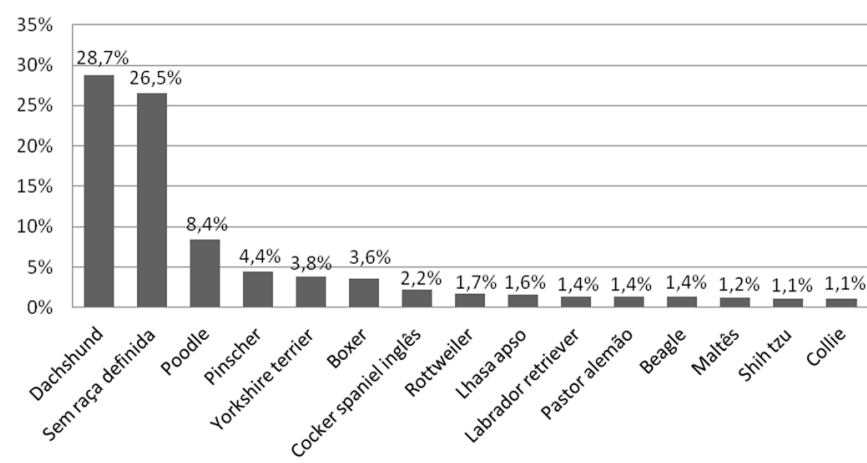

Fig.2. Distribuição das raças de cães $(n=1176)$ com doença neurológica atendidos no Serviço de Neurologia do Hospital Veterinário Universitário da Universidade Federal de Santa de 2006 a 2013.

Collie (1,1\%) (Fig.2). No Brasil, não se conhece dado populacional sobre doenças neurológicas em cães de acordo com as regiões geográficas do país (Fighera 2008). Porém, acredita-se que a dominância de cães de raças pequenas e condrodistróficas possa contribuir na explicação sobre a alta prevalência de doença degenerativa encontrada. 0 elevado número de cães sem raça definida pode estar relacionado ao fato de muitos proprietários adotarem animais de ONG's, prática comum em países da América Latina (Pellegrino et al. 2011). Por outro lado, Fluehmann et al. (2006) verificaram uma diminuição de cães de raça nos últimos anos, o que poderia explicar a maior prevalência de doenças do sistema nervoso afetando cães sem raça definida.

Dos 1.184 cães inclusos nesse estudo, o diagnóstico etiológico foi confirmado em 525 cães (44,4\%) e, em 659 $(55,6 \%)$, permaneceu como presuntivo. 0 diagnóstico presuntivo foi determinado pela exclusão das demais doenças listadas no diagnóstico diferencial e pelas características da suspeita clínica principal definidas na resenha, no histórico, na evolução clínica (aguda ou crônica, progressiva ou não progressiva) e na resposta ao tratamento (Fluehmann et al. 2006). Dos 1.184 cães, 147 (12,4\%) foram encaminhados para o Laboratório de Patologia Veterinária da UFSM para realização de necropsia. Desses, em 12 cães $(8,1 \%)$ não foram encontradas alterações no exame histopatológico, portanto, classificadas como diagnóstico presuntivo; o não estabelecimento do diagnóstico pode ser a ausência de lesões macroscópicas e histológicas nas estruturas analisadas (Fighera 2008). A quantidade relativamente elevada de diagnósticos presuntivos se deve provavelmente a não realização de exames complementares específicos, pela indisponibilidade de equipamentos sofisticados de imagem, como ressonância magnética, para confirmar ou excluir determinadas doenças ou da não realização de necropsia e exame histopatológico do sistema nervoso central, os quais são métodos importantes para concluir o diagnóstico de determinadas doenças, principalmente as inflamatórias de origem não infecciosa (Schatzberg 2010). Deve se ressaltar o papel do clínico veterinário em conscientizar os proprietários da importância da realização de necropsias, visto que, diversos animais desse estudo morreram durante o tratamento clínico e não foram submetidos a necropsia pela falta de notificação por parte dos proprietários. No estudo 
de Fluehmann et al. (2006), dos 4.497 cães, em 416 (9\%) não havia registro sobre o diagnóstico. Dos 4.081 cães, em 32\% o diagnóstico definitivo foi confirmado; em 33\% permaneceu o diagnóstico presuntivo e em $26 \%$ o diagnóstico foi obtido pelo exame histopatológico, essa última categoria não inclusa no presente estudo. Já Pellegrino et al. (2011) obtiveram um índice maior de diagnósticos concluídos (88\%) quando comparados aos presuntivos (12\%), provavelmente pela realização de exames complementares específicos, como histopatologia e diagnóstico de imagem avançado ( $24 \%$ de todos os casos).

Dos 1.184 cães avaliados, foi estabelecido diagnóstico neuroanatômico em todos os casos (Fig.3). Em 66,4\% dos casos ( $\mathrm{n}=786)$, a lesão ocorreu na medula espinhal, sendo a região T3-L3 a mais acometida $(n=484$ [40,9\%]), seguida de C1-C5 ( $\mathrm{n}=156$ [13,2\%]). Os segmentos C6-T2 e L4-S3 contribuíram com apenas 5,1\% e 6,3\% dos casos atendidos ( $\mathrm{n}=61$ e 74), respectivamente. Para as estruturas supratentoriais, $17,5 \%(n=207)$ envolvia a região tálamo-cortical e apenas $2,7 \%(n=32)$, o tronco encefálico. As doenças que afetaram mais de uma região (multifocal) representaram $7,8 \%$ dos casos ( $\mathrm{n}=93)$, seguido de $4,5 \%(\mathrm{n}=53)$ do sistema nervoso periférico e $1,7 \%(n=20)$ neuromusculares. Apenas $0,3 \%$ dos casos $(n=4)$ apresentaram alterações envolvendo o cerebelo.

Como demonstrado anteriormente, os segmentos da medula espinhal mais afetados foram T3-L3 (489 cães, [41,3\%]) e C1-C5 (160 cães [13,5\%]). Dos 1.184 cães que tinham diagnóstico de alteração nessas regiões, a doença do disco intervertebral (DDIV) foi a mais frequente (542 cães [45,8\%]), sendo 220 cães $(40,6 \%)$ com diagnóstico confirmado e 322 cães $(59,4 \%)$ com diagnóstico presuntivo. Dos cães com diagnóstico definitivo $(n=220), 91 \%$ foram confirmados por cirurgia e $9 \%$ por necropsia. Quanto à região neuroanatômica, dos 542 cães com DDIV, 69,4\% $(\mathrm{n}=376)$ apresentavam lesão entre T3-L3, corroborando com os achados de Dewey (2006) e Lorenz et al. (2011), que afirmam ser essa região a mais frequentemente afetada, seguida das regiões C1-C5 ( $\mathrm{n}=105$ [19,4\%]); C6-T2 $(n=29[5,3 \%]) ;$ L4-S3 $(n=26[4,8 \%]) ;$ e multifocal $(n=6$ $[1,1 \%])$. Dos animais em que constava a raça nos registros analisados (537 cães [99\%]), Dachshund (303 cães

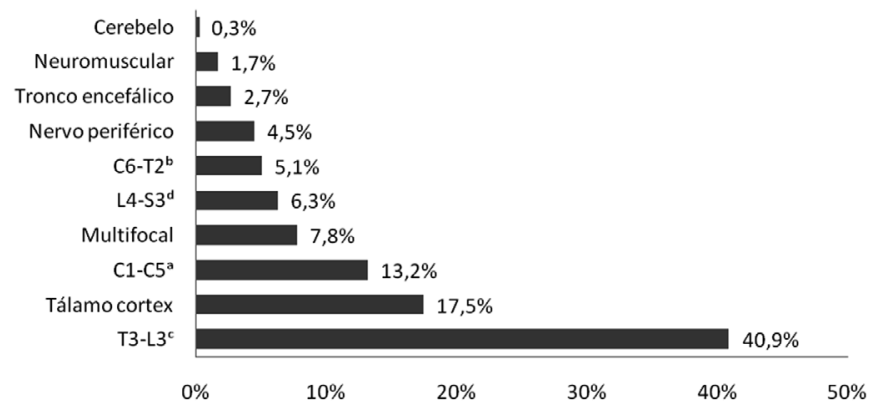

Fig.3. Distribuição de frequências dos grupos de diagnóstico neuroanatômico de acordo com os sinais clínicos $(n=1184)$ de cães atendidos no Serviço de Neurologia do Hospital Veterinário Universitário da Universidade Federal de Santa de 2006 a

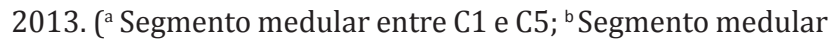
entre C6 e T2; ' Segmento medular entre T3 e L3; d Segmento medular entre L4 e S3)
[56,4\%]) foi a mais acometida, seguida pelos cães sem raça definida (101 cães [18,8\%]), Poodle (31 cães [5,7\%]), Beagle (12 cães [2,2\%]) e Cocker spaniel inglês (11 cães [2\%]), o que indica que cães de raças condrodistróficas são mais prodispostos que não condrodistróficas (Kranenburg et al. 2013, Smolders et al. 2013,). Neste estudo verificou-se que todos os casos de DDIV foram encontrados em cães com mais de 24 meses. Portanto, mesmo sendo Dachshund a raça mais acometida pela doença (Santos et al. 2011, Santos et al. 2012), ao suspeitá-la, deve se levar em consideração outros fatores, como a idade do paciente, já que o surgimento dos sinais clínicos ocorre em animais acima de dois anos de idade, mesmo que o processo degenerativo do disco para essa raça se inicie nos primeiros meses de vida (Smolders et al. 2013).

A doença com diagnóstico presuntivo mais frequente nesse estudo de também foi a DDIV ( $\mathrm{n}=322$ [48,9\%]). Diversos fatores, como a responsividade do paciente ao tratamento clínico, provavelmente influenciaram, principalmente para cães em grau I (somente dor à palpação epaxial) e II (ataxia proprioceptiva) de disfunção neurológica, ou devido a pacientes já apresentarem grau mais avançado (grau V [paraplegia com ausência de dor profunda]) durante um período maior que 48 horas, sendo assim não recomendado o tratamento cirúrgico (Lorenz et al. 2011) e, consequentemente, não confirmando o diagnóstico.

Nos cães desse estudo $(n=1184)$ foi possível distribuir a doença quanto à categoria pelos critérios do acrônimo DINAMIT-V (Fig.4). Assim como em outros estudos (Fluehmann et al. 2006, Pellegrino et al. 2011), essa classificação foi feita após o diagnóstico (definitivo ou presuntivo), ao contrário do que é empregado na rotina do Serviço de Neurologia da Instituição, cuja lista de diagnósticos diferenciais é montada após o exame clínico e neurológico (diagnóstico anatômico) e previamente à solicitação dos exames complementares. As categorias de doenças mais diagnosticadas foram a degenerativa ( $\mathrm{n}=581$ [49\%]), a inflamatória/infecciosa (n=197 [16,6\%]) e a traumática $(n=165$ $[13,9 \%])$. Resultados semelhantes foram encontrados no

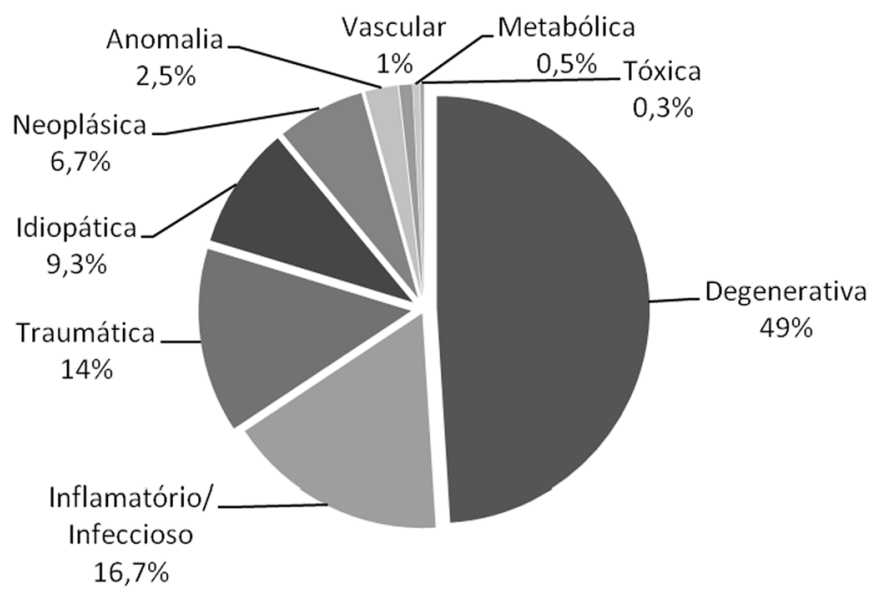

Fig.4. Distribuição percentual das categorias de enfermidades neurológicas estabelecidas de acordo com o acrônimo DINAMIT-V ( $\mathrm{n}=1184$ ) de cães com doença neurológica atendidos no Serviço de Neurologia do Hospital Veterinário Universitário da Universidade Federal de Santa de 2006 a 2013. 
estudo de Fluehmann et al. (2006), em que as categorias de enfermidades mais encontradas foram doenças degenerativas, inflamatórias/infecciosas e idiopáticas. Já no estudo realizado por Pellegrino et al. (2011), as doenças mais encontradas foram idiopática, congênitas e inflamatórias/ infecciosas. Segundo esses autores, a alta prevalência de pacientes com enfermidade idiopática pode estar relacionada ao diagnóstico de epilepsia, já que a doença pode ser primária ou idiopática.

As doenças inflamatórias/infecciosas constituíram a segunda categoria de enfermidades mais encontradas nesse estudo (197 cães [16,6\%]), sendo 60 cães $(30,5 \%)$ com diagnóstico definitivo e 137 (69,5\%) com diagnóstico presuntivo. Além das possíveis razões já mencionadas para o número elevado de diagnósticos presuntivos, uma situação encontrada e verificada durante essa pesquisa foi a não informação do óbito do animal por parte do proprietário e a impossibilidade da realização de necropsia. Dos 197 cães com enfermidades inflamatórias/infecciosas, 63\% (n=124) apresentaram diagnóstico de cinomose (30 casos com diagnóstico confirmado e 94 com presuntivo), sendo o diagnóstico confirmado pelo exame histopatológico. A cinomose foi a segunda doença mais encontrada nesse estudo e acredita-se que esse número poderia ser maior, visto que muitos pacientes atendidos no HVU com alterações neurológicas decorrentes de cinomose não são encaminhados ao SN pelos clínicos, principalmente quando os sinais neurológicos da doença são evidentes como, por exemplo, mioclonias (Dewey 2006). Dados epidemiológicos obtidos nesta Instituição em três estudos independentes (Headley \& Graça 2000, Silva et al. 2007, Fighera et al. 2008a), estimaram que a cinomose é responsável pela morte de aproximadamente $12 \%$ de todos os cães da rotina no HVU-UFSM encaminhados à necropsia. Vale ressaltar também que houve um alto número de diagnósticos presuntivos para essa categoria de doença e, provavelmente, para cinomose, numa proporção 1:3 (confirmado:presuntivo). Embora o diagnóstico para a cinomose possa ser clínico (Blixenkrone-Moller et al. 1993), principalmante quando há sinais clínicos e neurológicos evidentes, os autores deste trabalho salientam que outras afecções neurológicas podem confundir o clínico, principalmente quando os sinais neurológicos são inespecíficos (por exemplo; crises convulsivas e andar compulsivo), já que exames de auxílio ao diagnóstico, como análise de LCE e hemograma não permitem confirmar ou excluir a doença em questão. Portanto, ao suspeitar de doenças infecciosas, principalmente cinomose, se indica incluir outras enfermidades no diagnóstico diferencial e tentar "fugir" da regra "é cinomose".

Meningoencefalomielites não-infecciosas foram observadas em 23 cães (11\%); sete cães $(30,4 \%)$ com diagnóstico confirmado e $16(69,6 \%)$ com diagnóstico presuntivo, sendo a meningoencefalite granulomatosa, a meningoencefalite necrosante e a meningite-arterite imunomediada as mais frequentes. A etiologia, na maioria das vezes, pode ser autoimune (Dewey 2006), considerada uma causa comum e importante de distúrbios neurológicos em cães. A incidência dessas doenças varia de acordo com a região, contudo, sua prevalência não está claramente estabelecida e os sinais clínicos são semelhantes quando comparados a outras doenças inflamatórias, infecciosas ou não, sendo um desafio diagnóstico ante mortem para o clínico (Schatzberg 2010).

Na categoria de doença traumática no presente trabalho, o atropelamento por veículos automotivos foi a causa mais comum de trauma medular, como citado por Chai et al. (2008), Bruce et al. (2008) e Fighera et al. (2008). Dos 114 cães com trauma medular, 51 (45\%) foram decorrentes de acidentes automobilísticos. O local mais afetado foi o segmento T3-L3 ( $\mathrm{n}=67$ [58,8\%]), seguido de L4-S3 ( $n=33$ [29\%]), corroborando com os estudos de Bruce et al. (2008) e Bali et al. (2009) ao demostrarem que a maioria das lesões vertebrais traumáticas nos cães está localizada nos segmentos medulares supracitados. A alta prevalência de cães com traumas medulares observada na região do estudo provavelmente esteja relacionada ao elevado número de animais errantes, o fato de a Instituição estar próxima de rodovias de alto fluxo de veículos e de proprietários que omitem medidas de segurança durante passeios, como uso de guias e coleiras.

Em algumas situações, a classificação da doença em determinada categoria do DINAMIT V é suficiente para iniciar um tratamento adequado e correção do problema neurológico, mesmo quando não foi possível chegar ao diagnóstico definitivo (diagnóstico terapêutico). Deve se ressaltar que a utilização do acrônimo DINAMIT V é um método eficaz, que auxilia o clínico na busca pelos principais diagnósticos diferenciais relacionados aos sinais neurológicos presentes (Pellegrino et al. 2011). Associado a isso, deve se incluir sempre dados sobre idade, espécie, raça, progressão dos sinais (aguda e crônica), assimetria de sinais e presença ou ausência de dor, principalmente na coluna vertebral, na classificação em alguma das categorias do DINAMIT-V.

Os resultados desse trabalho demostraram que a formação de um banco de dados permite analisar a distribuição dos casos quanto à frequência de distúrbios neurológicos em relação a variáveis como categorias de doenças e regiões neuroanatômicas. Embora fossem incluídos os casos com diagnóstico presuntivo pode se verificar que determinadas doenças com sinais neurológicos diagnosticadas em outras regiões do país e do mundo não foram observadas nesse estudo. Com isso, não se deve restringir à abordagem diagnóstica de um determinado paciente, mas estender para a análise da distribuição regional (Fluehmann et al. 2006), o que fornece informações sobre as características das diferentes doenças neurológicas em diferentes regiões geográficas de um país.

Portanto, a relevância clínica desse estudo foi apresentar dados epidemiológicos das principais doenças neurológicas de cães atendidos na Região Central do Rio Grande do Sul, a fim de auxiliar os clínicos na elaboração de um plano diagnóstico, incluindo diagnóstico diferencial, bem como orientar na solicitação dos exames complementares de maneira mais criteriosa.

\section{CONCLUSÕES}

Com base nos resultados obtidos na avaliação das fichas neurológicas do Serviço de Neurologia do HVU-UFSM, pode 
se concluir que as doenças neurológicas em cães foram mais prevalentes em fêmeas.

Quanto à idade, cães com três a nove anos foram mais afetados e, quanto à raça, Dachshund e cães sem raça definida foram os mais acometidos.

As regiões neuroanatômicas mais afetadas foram a medula espinhal entre T3-L3 e C1-C5 e a tálamo-cortical.

A categoria mais diagnosticada de doenças neurológicas pelo acrônimo DINAMIT-V foi a degenerativa, seguida das doenças inflamatórias/infecciosas e traumáticas.

\section{REFERÊNCIAS}

Bagley R.S., Kornegay J.N., Page R.L. \& Thrall D.E. 1993. Central nervous system-oncology, p.2137-2166. In: Slatter D. (Ed.), Textbook of Small Animal Surgery. $2^{\text {nd }}$ ed. W.B. Saunders, Philadelphia.

Bagley R.S., Gavin P.R., Moore M.P., Silver G.M., Harrington M.L. \& Connors R.L. 1999. Clinical signs associated with brain tumors in dogs: 97 cases (1992-1997). J. Am. Vet. Med. Assoc. 215(6):818-819.

Bali M.S., Lang J., Jaggy A., Spreng D., Doherr M.G. \& Forterre F. 2009. Comparative study of vertebral fractures and luxations in dogs and cats. Vet. Comp. Orthop. Traumatol. 22(1):47-53.

Blixenkrone-Moller M., Svansson V., Have P., Orvell C., Apple M., Pedersen I.R., Dietz H.H. \& Henriksen P. 1993. Study on manifestations of canine distemper virus infection in an urban dog population. Vet. Microbiol. 37(1/2):163-173.

Bradshaw J.M., Pearson G.R. \& Gruffyd-Jones T.J. 2004. A retrospective study of 286 cases of neurological disorders of the cat. J. Comp. Pathol. 131(2/3):112-120.

Brisson B.A. 2010. Vertebral intervertebral disc disease in dogs. Vet. Clin. North Am., Small Anim. Pract. 40(5):829-858.

Bruce C.W., Brisson B.A. \& Gyselinck K. 2008. Spinal fracture and luxation in dogs and cats: a retrospective evaluation of 95 cases. Vet. Comp. Orthop. Traumatol. 21(3):280-284.

Chai O., Johnston D.E. \& Shamir M.H. 2008. Bite wounds involving the spine: characteristics, therapy and outcome in seven cases. Vet J. 175(2):259-265.

Da Costa R.C. \& Moore S.A. 2010. Differential diagnosis of spinal diseases. Vet. Clin. North Am., Small Anim. Pract. 40(5):755-763.

Dewey C.W. 2006. Neurologia de Cães e Gatos. Roca, São Paulo.

Fighera R.A. 2008. Causas de morte e razões para eutanásia em cães. Tese de Doutorado, Curso de Pós-Graduação em Medicina Veterinária, Universidade Federal de Santa Maria, Santa Maria, RS. 28p.

Fighera R.A., Silva M.C., Souza T.M., Brum J.S., Kommers D., Graça D.L., Irigoyen L.F. \& Barros C.S.L. 2008. Aspectos patológicos de 155 casos fatais de cães atropelados por veículos automotivos. Ciência Rural 38(5):1375-1380.

Fighera R.A., Souza T.M., Silva M.C., Brum J.S., Graça D.L., Kommers G.D., Irigoyen L.F. \& Barros C.S.L. 2008a. Causas de morte e razões para eutanásia de cães da Mesorregião do Centro Ocidental Rio-Grandense (1965-2004). Pesq. Vet. Bras. 28(4):223-230.

Fluehmann G., Doherr M.G. \& Jaggy A. 2006. Canine neurological diseases in a referral hospital population between 1989 and 2000 in Switzerland. J. Small Anim. Pract. 47(10):582-587.
Foster E.S., Carrillo J.M. \& Patnaik A.K. 1988. Clinical signs of tumors affecting the rostral cerebrum in 43 dogs. J. Vet. Intern. Med. 2(2):71-74.

Garosi L., McConnel J.E., Platt S.R., Barone G., Baron J.C., De Lahunta A. \& Schatzberg S.J. 2005. Results of diagnostic investigations and long-term outcome of 33 dogs with brain infarction (2000-2004). J. Vet. Intern. Med. 19(5):725-773.

Hayes H.M., Priester Jr W.A. \& Pendergrass T.W. 1975. Occurrence of nervous-tissue tumors in cattle, horses, cats and dogs. Int. J. Cancer 15(1):39-47.

Headley S.A. \& Graça D.L. 2000. Canine distemper: epidemiological findings of 250 cases. Braz. J. Vet. Res. Anim. Sci. 37(2):136-140.

Heidner G.L., Kornegay J.N., Page R.L., Dodge R.K. \& Thrall D.E. 1991. Analysis of survival in a retrospective study of 86 dogs with brain tumors. J. Vet. Intern. Med. 5(4):219-226.

Kipperman B.S., Feldman E.C., Dybdal N.O. \& Nelson R.W. 1992. Pituitary tumor size, neurologic signs, and relation to endocrine tests results in dogs with pituitary-dependent hyperadrenocorticism: 43 cases (19801990). J. Am. Vet. Med. Assoc. 201(5):762-767.

Kranenburg H.J.C., Grinwis G.C.M., Bergknut N., Gahrmann N., Voorhout G., Hazewinkel H.A.W. \& Meij B.P. 2013. Intervertebral disc disease in dogs - Part 2: Comparison of clinical, magnetic resonance imaging, and histological findings in 74 surgically treated dogs. Vet. J. 195(2):164-171.

Lorenz M.D., Coates J.R. \& Kent M. 2011. Handbook of Veterinary Neurology. $5^{\text {th }}$ ed. W.B. Saunders, Philadelphia, PA.

Marioni-Henry K., Vite C.H., Newton A.L. \& Van Winkle T.J. 2004. Prevalence of diseases of the spinal cord of cats. J. Vet. Intern. Med. 18(6):851858.

Marioni-Henry K., Van Winkle T.J., Smith S.H. \& Vite C.H. 2008. Tumors affecting the spinal cord of cats: 85 cases (1980-2005). J. Am. Vet. Med. Assoc. 232(2):237-243.

Pellegrino F.C., Pacheco E.L. \& Vazzoler M.L. 2011. Caracterización de los trastornos neurológicos en los perros: 1652 casos (marzo 2008-junio 2010). Parte I. Revta. Argent. Neurol. Vet. 2(1):78-96.

Santos R.P., Mazzanti A., Beckmann D.V., Berté L., Ripplinger A., Neto D.P. \& Baumhardt R. 2011. Recuperação funcional em cães com doença do disco intervertebral toracolombar sem percepção à dor profunda: 37 casos (2002-2010). Pesq. Vet. Bras. 31(4):345-349.

Santos R.P., Beckmann D.V., Aiello G., Berté L., Ripplinger A., Neto D.P. \& Mazzanti A. 2012. Recuperação funcional de cães paraplégicos com doença do disco intervertebral toracolombar sem percepção à dor profunda, submetidos ao tratamento cirúrgico: 15 casos (2006-2010). Pesq. Vet. Bras. 32(3):243-246.

Schatzberg S.J. 2010. Idiopathic granulomatous and necrotizing inflammatory disorders of the canine central nervous system. Vet. Clin. Small Anim. 40(1):101-120.

Silva M.C., Fighera R.A., Brum J.S., Graça D.L., Kommers G.D., Irigoyen L.F. \& Barros C.S.L. 2007. Aspectos clinicopatológicos de 620 casos neurológicos de cinomose em cães. Pesq. Vet. Bras. 27(5):215-220.

Smolders L.A., Bergknut N., Grinwis G.C.M., Hagman R., Lagerstedt A.S., Hazewinkel H.A.W., Tryfonidou M.A. \& Meij B.P. 2013. Intervertebral disc degeneration in the dog. Part 2: Chondrodystrophic and non-chondrodystrophic breeds. Vet. J. 195(3):292-299.

Snyder J.M., Shofer F.S., Van Winkle T.J. \& Massicotte C. 2006. Canine intracranial primary neoplasia: 173 cases (1986-2003). J. Vet. Intern. Med. 20(3):669-675. 